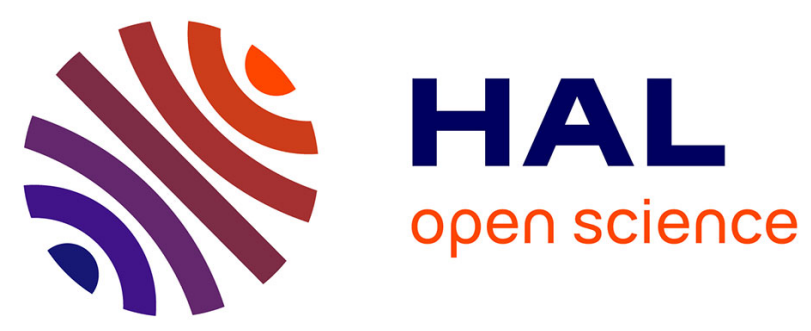

\title{
Multi-Spherical Diffusion MRI: Exploring Diffusion Time Using Signal Sparsity
}

\author{
Rutger H.J. Fick, Alexandra Petiet, Mathieu Santin, Anne-Charlotte \\ Philippe, Stéphane Lehéricy, Rachid Deriche, Demian Wassermann
}

\section{- To cite this version:}

Rutger H.J. Fick, Alexandra Petiet, Mathieu Santin, Anne-Charlotte Philippe, Stéphane Lehéricy, et al.. Multi-Spherical Diffusion MRI: Exploring Diffusion Time Using Signal Sparsity. MICCAI 2016 Workshop on Computational Diffusion MRI (CDMRI'16), Oct 2016, Athènes, Greece. hal-01354985

\author{
HAL Id: hal-01354985 \\ https://hal.science/hal-01354985
}

Submitted on 22 Aug 2016

HAL is a multi-disciplinary open access archive for the deposit and dissemination of scientific research documents, whether they are published or not. The documents may come from teaching and research institutions in France or abroad, or from public or private research centers.
L'archive ouverte pluridisciplinaire HAL, est destinée au dépôt et à la diffusion de documents scientifiques de niveau recherche, publiés ou non, émanant des établissements d'enseignement et de recherche français ou étrangers, des laboratoires publics ou privés. 


\title{
Multi-Spherical Diffusion MRI: Exploring Diffusion Time Using Signal Sparsity
}

\author{
Rutger H.J. Fick ${ }^{1}$, Alexandra Petiet ${ }^{2}$, Mathieu Santin ${ }^{2}$, Anne-Charlotte \\ Philippe $^{2}$, Stephane Lehericy ${ }^{2}$, Rachid Deriche ${ }^{1}$, and Demian Wassermann ${ }^{1}$ \\ 1 Université Côte d'Azur, Inria, France \\ 2 CENIR, Institut du Cerveau et de la Moelle épineère, Paris, France
}

\begin{abstract}
Effective representation of the diffusion signal's dependence on diffusion time is a sought-after, yet still unsolved, challenge in diffusion MRI (dMRI). We propose a functional basis approach that is specifically designed to represent the dMRI signal in this four-dimensional spacevarying over gradient strength, direction and diffusion time. In particular, we provide regularization tools imposing signal sparsity and signal smoothness to drastically reduce the number of measurements we need to probe the properties of this multi-spherical space. We illustrate a novel application of our approach, which is the estimation of time-dependent q-space indices, on both synthetic data generated using Monte-Carlo simulations and in vivo data acquired from a C57Bl6 wild-type mouse. In both cases, we find that our regularization approach stabilizes the signal fit and index estimation as we remove samples, which may bring multi-spherical diffusion MRI within the reach of clinical application.
\end{abstract}

\section{Introduction}

Effective representation of the diffusion signal's dependence on diffusion time is a sought-after, yet still unsolved challenge in diffusion MRI (dMRI). Recent literature is increasingly emphasizing the need for such a representation, where accounting for the diffusion time dependence of the extra-axonal diffusion signal $[1,2]$ has already resulted in a more accurate estimation of the axon density and diameter [3]. To measure the four-dimensional dMRI signal it is necessary to go beyond a multi-shell q-space acquisition - which only varies gradient strength and direction - and also vary the diffusion time. This multi-spherical acquisition is hardly feasible in a clinical setting due to a large number of sample points in this four-dimensional space-time framework.

To reduce the number of required samples, we propose to leverage the recently proposed representation of the multi-spherical signal in terms of an orthogonal functional basis inspired by Fick et al. [4]. Particularly, we will show that the multi-spherical dMRI signal is sparse when represented in terms of this basis. Different sparse signal reconstruction frameworks, e.g. [5,6], have shown that signal sparsity allows for a significant reduction in the number of acquired samples. Furthermore, sparse signal reconstruction has been successfully used in different dMRI protocols, see e.g. [7-10]. However, to the best of our knowledge, 
we are the first to facilitate microstructural measurements by leveraging the sparsity of the spatial and temporal dMRI signal using a novel functional basis. We demonstrate that we are able to reduce the number of required samples for a multi-spherical dMRI acquisition and derive time-dependent microstructural features on both simulated data and in-vivo mouse data.

This paper is structured as follows: first, we present the theory behind our estimation method in Section 2. We then describe our methods of generating insilico multi-spherical data and the parameters of our in vivo dMRI acquisition of C57Bl6 wild-type mouse in Section 3. In section 4 we then show the results of our method, we discuss our findings and present our conclusions in Section 5.

\section{Theory}

We first provide the relation between the measured multi-spherical diffusion signal and the four-dimensional ensemble average propagator (EAP) in section 2.1. We then explain the properties that we would like our multi-spherical representation to have, and provide the details on the functional basis representation and regularization which are used to impose the desired properties in Section 2.2.

\subsection{The Four-Dimensional Ensemble Average Propagator}

In dMRI, the EAP describes the probability density that a spin diffuses a certain distance in a given diffusion time. The EAP is estimated by obtaining diffusionweighted images (DWIs). A DWI is obtained by applying two sensitizing diffusion gradients of pulse length $\delta$ to the tissue, separated by separation time $\Delta$. The resulting signal is 'weighted' by the average particle movements along the applied gradient direction. When these gradients are considered infinitely short $(\delta \rightarrow 0)$, which can only be approximated in practice, the relation between the measured signal $S(\mathbf{q}, \tau)$ and the EAP $P(\mathbf{r} ; \tau)$ is given by a Fourier transform [11] as

$$
E(\mathbf{q}, \tau)=\int_{\mathbb{R}^{3}} P(\mathbf{R} ; \tau) e^{-2 \pi i \mathbf{q} \cdot \mathbf{r}} d \mathbf{R} \quad \text { with } \quad \mathbf{q}=\frac{\gamma \delta \mathbf{G}}{2 \pi} \text { and } \tau=\Delta-\delta / 3,
$$

where $E(\mathbf{q}, \tau)=S(\mathbf{q}, \tau) / S_{0}$ is the normalized signal attenuation measured at diffusion encoding position $\mathbf{q}$, and $S_{0}$ is the baseline image acquired without diffusion sensitization $(q=0)$. We denote $q=|\mathbf{q}|, \mathbf{q}=q \mathbf{u}$ and $\mathbf{R}=R \mathbf{r}$, where $\mathbf{u}$ and $\mathbf{r}$ are $3 \mathrm{D}$ unit vectors and $q, R \in \mathbb{R}^{+}$. The wave vector $\mathbf{q}$ on the right side of Eq. (1) is related to pulse length $\delta$, nuclear gyromagnetic ratio $\gamma$ and the applied diffusion gradient vector $\mathbf{G}$.

The four-dimensional EAP has boundary conditions with respect to $\{\mathbf{q}, \tau\}$ :

$-\{\mathbf{q}, \tau=0\}$ : When $\tau=0$ the spins have no time to diffuse and the EAP is a spike function at the origin, i.e, $P(\mathbf{R} ; \tau=0)=\delta(R)$. Following Eq. (1), the signal attenuation will not attenuate for any value of $\mathbf{q}$, i.e., $E(\mathbf{q}, \tau=0)=1$. 
- $\left\{\mathbf{q}, \lim _{\tau \rightarrow \infty}\right\}:$ When $\lim _{\tau \rightarrow \infty} E(\mathbf{q}, \tau)$ the signal attenuation is in the long diffusion time limit and only signal contributions from restricted compartments remain [16]. In this case, given infinite gradient strength and some assumptions on tissue composition [12,13], q-space indices such as the Return-ToAxis Probability (RTAP) are related to the mean apparent axon diameter.

$-\{\mathbf{q}=0, \tau\}$ : When $\mathbf{q}=0$ there is no diffusion sensitization so $E(\mathbf{q}=0, \tau)=1$. With Fourier relationship in Eq. (1), this point also corresponds to the zeroth harmonic of the EAP, which as a probability density integrates to one.

$-\left\{\lim _{\mathbf{q} \rightarrow \infty}, \tau\right\}: \lim _{\mathbf{q} \rightarrow \infty} E(\mathbf{q}, \tau)=0$, as even an infinitesimally small spin movement will attenuate the signal completely.

\subsection{Multi-Spherical Signal Representation}

In dMRI, functional basis approaches have been used to efficiently represent the diffusion signal with little assumptions on its shape. Following this methodology, we represent the measured multi-spherical signal $E(\mathbf{q}, \tau)$ in terms of a continuous functional basis $\hat{E}(\mathbf{q}, \tau ; \mathbf{c})$, where the signal is now represented in terms of coefficients $\mathbf{c} \in \mathbb{R}^{N_{c}}$. An effective representation $\hat{E}(\mathbf{q}, \tau ; \mathbf{c})$ should be able to

1. closely approximate the measured multi-spherical dMRI signal,

2. smoothly interpolate between and outside the measured $\{\mathbf{q}, \tau\}$ points,

3. have a sparse representation in $\mathbf{c}$,

4. be able to reconstruct the EAP from the fitted signal.

Requirements 1-3 are described in Eq. (2), while the fourth will follow by choosing a functional basis that is also a Fourier basis.

$$
\begin{aligned}
& \operatorname{argmin}_{\mathbf{c}} \overbrace{\iint[E(\mathbf{q}, \tau)-\hat{E}(\mathbf{q}, \tau ; \mathbf{c})]^{2} d \mathbf{q} d \tau}^{(1) \text { Data Fidelity }}+\overbrace{\iint\left[\nabla^{2} \hat{E}(\mathbf{q}, \tau ; \mathbf{c})\right]^{2} d \mathbf{q} d \tau}^{(2) \text { Smoothness }}+\overbrace{\|\mathbf{c}\|_{1}}^{(3) \text { Sparsity }} \\
& \text { subject to } E(0, \tau ; \mathbf{c})=1, E(\mathbf{q}, 0 ; \mathbf{c})=1, \quad \hat{P}(\mathbf{R}, \tau ; \mathbf{c})=\operatorname{IFT}(\hat{E}(\mathbf{q}, \tau ; \mathbf{c}))
\end{aligned}
$$

Note that the integrals over three-dimensional $\mathbf{q}$ have limits $[-\infty, \infty]$ and those over $\tau$ have limits $[0, \infty]$. As stated in Section 2.1, the boundary constraints are important to respect the Fourier relationship between the fitted signal attenuation and the EAP.

Functional Basis Signal Representation We represent the multi-spherical signal using an orthogonal basis that allows for the implementation of all our previously stated requirements. As we assume an infinitely short gradient pulse $(\delta \rightarrow 0)$, we follow Callaghan et al.'s description of time-dependent diffusion in pores and assume separability in the dependence of the dMRI signal to $\mathbf{q}$ and $\tau[16]$. Following this hypothesis, we can independently choose any representation 
for these two spaces. We represent the combined space $\hat{E}(\mathbf{q}, \tau ; \mathbf{c})$ using the crossproduct between the spatial basis $\Phi(\mathbf{q})$ and temporal basis $T(\tau)$ as

$$
\hat{E}(\mathbf{q}, \tau ; \mathbf{c})=\sum_{i}^{N_{\mathbf{q}}} \sum_{k}^{N_{\tau}} \mathbf{c}_{i k} \Phi_{i}(\mathbf{q}) T_{k}(\tau),
$$

where $N_{\mathbf{q}}$ and $N_{\tau}$ are the maximum expansion order of each basis and $\mathbf{c}_{i k}$ weights the contribution of the $i k^{t h}$ basis function to $\hat{E}(\mathbf{q}, \tau ; \mathbf{c})$.

A plethora of functional bases to represent $\mathbf{q}$ have been proposed, e.g. [8, 9, 12, 14]. Of these bases, we use the Mean Apparent Propagator (MAP) basis [12] as it neatly fulfills all four previously stated requirements; (1) being an orthogonal basis, it can accurately represent any signal over $\mathbf{q}$ using few coefficients; (2) it allows to impose smoothness using analytic Laplacian regularization [13]; (3) the isotropic MAP implementation was successfully used to obtain sparse signal representation [8] and (4) MAP is a Fourier basis. It is worth noting that this basis is different than Fick et al.'s basis, who used the isotropic implementation (3D-SHORE) to represent $\mathbf{q}[4]$.

MAP's signal basis is a product of three orthogonal Simple Harmonic Oscillatorbased Reconstruction and Estimation (SHORE) functions $\phi_{n}(u)$ [15]:

$$
\begin{array}{r}
\Phi_{N(i)}(\mathbf{q}, \mathbf{A})=\phi_{n_{1}}\left(q_{x}, u_{x}\right) \phi_{n_{2}}\left(q_{y}, u_{y}\right) \phi_{n_{3}}\left(q_{z}, u_{z}\right) \\
\text { with } \phi_{n}(q, u)=\frac{i^{-n}}{\sqrt{2^{n} n !}} e^{-2 \pi^{2} q^{2} u^{2}} H_{n}(2 \pi u q)
\end{array}
$$

with its Fourier transform, the EAP basis as

$$
\begin{aligned}
& \Psi_{N(i)}(\mathbf{R}, \mathbf{A})=\psi_{n_{1}}\left(R_{x}, u_{x}\right) \psi_{n_{2}}\left(R_{y}, u_{y}\right) \psi_{n_{3}}\left(R_{z}, u_{z}\right) \\
& \text { with } \psi_{n}(R, u)=\frac{1}{\sqrt{2^{n+1} \pi n !} u} e^{-R^{2} /\left(2 u^{2}\right)} H_{n}(R / u)
\end{aligned}
$$

where $H$ is a physicist's Hermite polynomial of order $n$ and $u$ is a data-dependent scale factor. As in MAP [12], before fitting, the data is rotated such that the DTI eigenvectors are aligned with the coordinate axis and we can use the datadependent scaling matrix $\mathbf{A}=\operatorname{Diag}\left(u_{x}^{2}, u_{y}^{2}, u_{z}^{2}\right)$ to scale the MAP basis functions according to the anisotropy of the data. The zeroth order is a purely Gaussian function while higher orders use the Hermite to correct this approximation to the true shape of the data. For a given radial order $N_{\text {rad }}$ the number of coefficients is $N_{\mathbf{q}}=\left(N_{\mathrm{rad}}+2\right)\left(N_{\mathrm{rad}}+4\right)\left(2 N_{\mathrm{rad}}+3\right) / 24$.

Our functional basis to describe $\tau$ was introduced in Fick et al. [4]. As a limiting case the diffusion signal dependence on $\tau$ is exponential for pure Gaussian diffusion and constant for diffusion in restricted geometries. To represent $\tau$ we, therefore, choose a product of the negative exponential and a Laguerre polynomial $L$, which together form an orthogonal basis over $\tau$

$$
T_{p}\left(\tau, u_{t}\right)=\exp \left(-u_{t} \tau / 2\right) L_{o}\left(u_{t} \tau\right)
$$


with basis order $p$ and temporal scaling factor $u_{t}$. The zeroth order is a pure exponential function and higher orders use the Laguerre polynomials to correct this approximation to the true shape of the signal.

For the rest of this work we will linearize the ordering of our multi-spherical basis such that we use one basis index $i$ with notation

$$
\hat{E}(\mathbf{q}, \tau ; \mathbf{c})=\sum_{i}^{N_{\mathbf{c}}} \mathbf{c}_{i} \Xi_{i}\left(\mathbf{q}, \tau, \mathbf{A}, u_{t}\right)=\sum_{i}^{N_{\mathbf{c}}} \mathbf{c}_{i} \Phi_{N(i)}(\mathbf{q}, \mathbf{A}) T_{p(i)}\left(\tau, u_{t}\right)
$$

where the total number of fitted coefficients is $N_{\mathbf{c}}=\left(N_{\tau}+1\right)\left(N_{\mathbf{q}}+2\right)\left(N_{\mathbf{q}}+\right.$ $4)\left(2 N_{\mathbf{q}}+3\right) / 24$. Using this notation, the fitted signal $\hat{E}(\mathbf{q}, \tau ; \mathbf{c})$ in the Data Fidelity term in Eq. (2), with measured signal $\mathbf{y} \in \mathbb{R}^{N_{\mathbf{y}}}$ and $N_{\mathbf{y}}$ the number of samples, can be represented as $\hat{\mathbf{y}}=\Phi \mathbf{c}$ with $\Phi \in \mathrm{R}^{N_{\mathbf{y}} \times N_{\mathbf{c}}}$ with values $\Phi_{i j}=$ $\Xi_{j}\left(\mathbf{q}_{i}, \tau_{i}, \mathbf{A}, u_{t}\right)$.

The multi-spherical EAP can be reconstructed using MAP's Fourier properties [12]. The Fourier transform only concerns the $\mathbf{q}$-space, so the EAP is found simply by switching $\Phi(\mathbf{q}, \mathbf{A})$ in Eq. (7) by its Fourier transform in Eq. (5).

Analytic Laplacian Regularization We impose smoothness in the multispherical signal reconstruction by using the squared norm of the Laplacian of the reconstructed signal as a regularizer. We define the Smoothness term in Eq. (2) as Laplacian functional $U(\mathbf{c})$ as

$$
U(\mathbf{c})=\iint\left[\nabla^{2} \hat{E}(\mathbf{q}, \tau ; \mathbf{c})\right]^{2} d \mathbf{q} d \tau
$$

where, due to our choice of basis, the Laplacian of the reconstructed signal can be estimated as $\nabla^{2} E_{\mathbf{c}}(\mathbf{q}, \tau)=\sum_{i} c_{i} \nabla^{2} \Xi_{i}\left(\mathbf{q}, \tau, \mathbf{A}, u_{t}\right)$. Eq. (8) can be further rewritted in quadratic form as $U(\mathbf{c})=\mathbf{c}^{\mathrm{T}} \mathbf{U} \mathbf{c}$ with

$$
\mathbf{U}_{i k}=\iint \nabla^{2} \Xi_{i}\left(\mathbf{q}, \tau, \mathbf{A}, u_{t}\right) \cdot \nabla^{2} \Xi_{k}\left(\mathbf{q}, \tau, \mathbf{A}, u_{t}\right) d \mathbf{q} d \tau
$$

where the subscript $i k$ indicates the $i k^{t h}$ position in the regularization matrix. We use the orthogonality of the basis functions (standard inner product on $[0, \infty]$ ) to compute the values of the regularization matrix to a closed form depending only on the basis orders and scale factors. We provide $\mathbf{U}$ in Appendix A.

Coefficient Estimation from Multi-Spherical Data We represent the multispherical signal $E(\mathbf{q}, \tau)$ in terms of a sparse coefficient vector $\mathbf{c}$ as $\mathbf{y}=\Phi \mathbf{c}+\epsilon$ with $\Phi$ the observation matrix, $\mathbf{y}$ the signal values and $\epsilon$ the acquisition noise. We frame the numerical implementation of our approach in the same way as we did continuously in Eq. (2):

$$
\begin{aligned}
& \underset{\mathbf{a r g m i n}}{\mathbf{c}} \overbrace{\|\mathbf{y}-\Phi \mathbf{c}\|_{2}}^{(1) \text { Data Fidelity }}+\overbrace{\beta\left\|\mathbf{c}^{T} \mathbf{U} \mathbf{c}\right\|_{2}}^{(2) \text { Smoothness }}+\overbrace{\alpha\|\mathbf{c}\|_{1}}^{(3) \text { Sparsity }} \\
& \text { subject to } \Phi^{\text {constraints }} \mathbf{c}=\mathbf{1}
\end{aligned}
$$


where we described the Data Fidelity and Smoothness term in Section 2.2, and the Sparsity term and constraints are imposed by framing our problem as a convex optimization using the open-source package CVXPY [17]. We find optimal values for regularization weights $\alpha$ and $\beta$ using cross-validation and implemented the surrounding code infrastructure inside the DiPy framework [18].

\subsection{Estimation of $\tau$-dependent q-space Indices}

Once coefficients c are known, our basis allows us to freely explore, for any diffusion time, all previously proposed scalar metrics for the three-dimensional EAP [12,13], also known as $\mathbf{q}$-space indices. We can do this because our basis reduces to the MAP basis when the temporal basis is evaluated for a particular diffusion time. In this work we illustrate this using the $\tau$-dependent Return-ToOrigin Probability (RTOP) and Mean Squared Displacement (MSD):

$$
M S D(\tau) \triangleq \int_{\mathbb{R}^{3}} \hat{P}(\mathbf{R}, \tau ; \mathbf{c}) \mathbf{R}^{2} d \mathbf{R} \quad \text { and } \quad R T O P(\tau) \triangleq \hat{P}(0, \tau ; \mathbf{c})
$$

\section{Data Set Specification}

Acquistion Scheme We will use the same acquisition scheme for both our synthetic and in vivo mouse experiments. An illustration of this scheme is given in Fig. 1. We acquire 32 different "shells" with 21 uniformly spread DWIs and one b0 each using pulse duration $\delta=5 \mathrm{~ms}$. Over these shells, we measure four equispaced " $\tau$-shells" $\Delta=\{8.7,12.2,15.8,19.4\} \mathrm{ms}$ and eight approximately equispaced "gradient shells" between $\{50-520\} \mathrm{mT} / \mathrm{m}$. The minimum b-value is $b_{\min }=48 \mathrm{~s} / \mathrm{mm}^{2}$ and maximum b-value is $b_{\max }=8590 \mathrm{~s} / \mathrm{mm}^{2}$.

In Silico Data Sets with Camino We use Camino [19] to reproduce diffusion signals originating from tissues containing realistic axon diameter distributions and packings. As we illustrate in Table 1, we use 5 gamma distributions from Aboitiz et al. [20] and 6 from Lamantia et al. [21]. Similarly as in Alexander et al. [22], we simulate the overall diffusion signal from these 11 distributions from the same distributions with doubled axonal diameters and two different packing densities, resulting in a total of 44 distributions.

Mouse acquisition data A spin echo sequence was acquired from a C57Bl6 wild-type mouse on an 11.7 Tesla Bruker scanner. The data consists of $96 \times$ $160 \times 12$ voxels of size $110 \times 110 \times 500 \mu \mathrm{m}$. We manually created a brain mask and corrected the data from eddy currents and motion artifacts using FSL's eddy_correct [23]. We then drew a region of interest consisting of 72 voxels in the middle slice in the corpus callosum, see Fig. 4, where we know the tissue is reasonably coherent.

\section{Experiments and Results}

Radial and Temporal Order Fitting In this noiseless experiment, we find the optimal choice of radial and temporal order to accurately fit the diffusion signal 


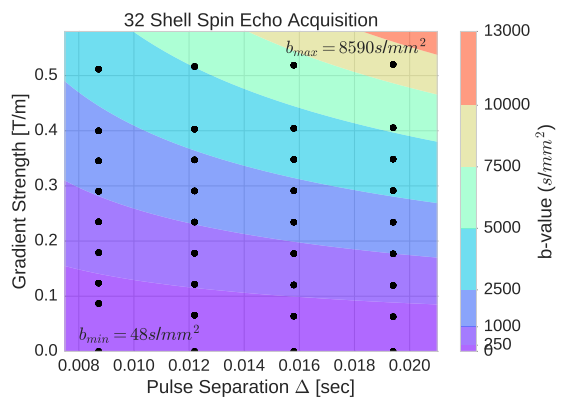

Fig. 1: Acquisition scheme for multispherical acquisition. Every dot represents a shell with 21 DWIs and one b0 image. The contours represent b-value isolines, whose values are given in the colorbar.
Table 1: Simulated Gamma distributions, sorted by mean axonal diameter $\langle D\rangle$.

\begin{tabular}{llll}
\hline Origin & \multicolumn{2}{l}{ shape $(\alpha)$ scale $(\beta)$} & $\langle D\rangle[\mu m]$ \\
\hline Aboitiz & 3.2734 & $2.4563 \mathrm{e}-07$ & 1.60 \\
Aboitiz & 2.8771 & $2.4932 \mathrm{e}-07$ & 1.43 \\
Aboitiz & 4.8184 & $1.3008 \mathrm{e}-07$ & 1.25 \\
Aboitiz & 3.5027 & $1.6331 \mathrm{e}-07$ & 1.14 \\
Aboitiz & 5.3316 & $1.0242 \mathrm{e}-07$ & 1.09 \\
Lamantia & 5.2051 & $1.0227 \mathrm{e}-07$ & 1.06 \\
Lamantia & 5.2357 & $9.3946 \mathrm{e}-08$ & 0.98 \\
Lamantia 10.1960 & $3.6983 \mathrm{e}-08$ & 0.75 \\
Lamantia 8.5358 & $3.7369 \mathrm{e}-08$ & 0.64 \\
Lamantia & 5.9242 & $5.3249 \mathrm{e}-08$ & 0.63 \\
Lamantia & 16.2750 & $1.4282 \mathrm{e}-08$ & 0.46
\end{tabular}

with the lowest number of coefficients. We fit our multi-spherical basis to the Camino data using different radial and temporal orders and calculate the mean squared error (MSE) of the fitted signal to the original signal. We show the result in Fig. 2a. We find that the mean absolute error of the signal over all distributions falls below $1 \%$ at a radial order of 6 and temporal order of 2 , resulting in 150 coefficients. We will use this combination in our next experiments.

Comparison with DTI Approximation In Fig. 2b we compare the MSE of fitting DTI, the basis of Fick et al. [4] and our multi-spherical approach to subsets of the noiseless data with increasing maximum b-values. As the maximum b-value increases, data with higher gradients strengths and diffusion times are included (see Fig 1). Our approach fits diffusion restriction over $\mathbf{q}$ and $\tau$ best of the three methods regardless of b-value.

Multi-spherical Signal Reconstruction and q-space Index Estimation To reduce the number of measurements, we regularize the basis fitting with a combination of imposing smoothness in the fitted signal and sparsity in the basis coefficients. To study its effectiveness, we first add Rician noise to the Camino data such that the signal-to-noise (SNR)-ratio is 20. We then randomly subsample, fit and then recover the data from our model and estimate the MSE with the noiseless data. The experiment for every chosen number of samples is repeated 50 times for all 44 voxels with each a different noise instance. The result can be seen in Fig. 3a, where our combined approach (yellow) has the lowest MSE, followed by using only the Laplacian (green) and the worst is least squares (red). We also show the effects of using between 600 samples (green) and 100 samples (blue) on the estimation of the Mean Squared Displacement (MSD) and the q-space index Return-To-Origin probability (RTOP) in Figs. 3b and 3c. We see that MSD increases as time increases, while its profile does not change much until the profile flattens for 100 samples. In contrast, we see that RTOP 


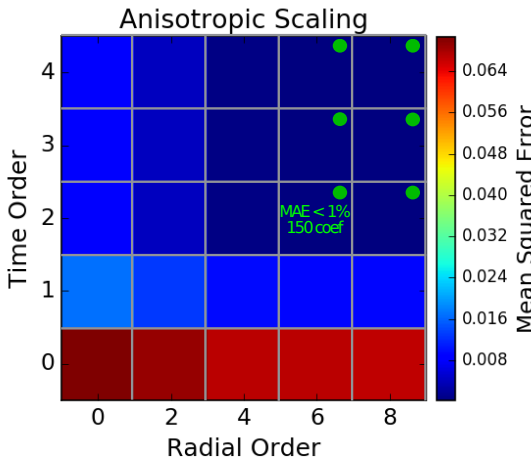

(a) MSE over basis order

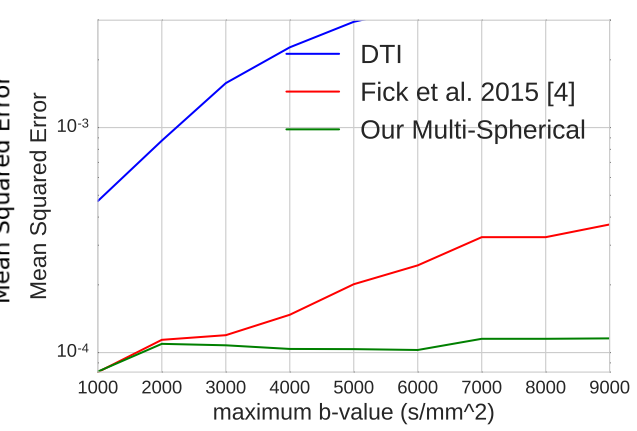

(b) DTI versus Multi-Spherical

Fig. 2: (a) Noise free fitting of Camino data set using different radial and time orders using our multi-spherical basis. The color intensity shows the mean squared error and the green dots indicate orders for which the mean absolute error of the reconstruction is smaller than $1 \%$ of the b0 value. (b) Comparison of the fitting error between DTI, the approach of Fick et al. [4] and our multi-spherical approach over maximum b-value.

decreases over time and as the number of samples reduces, the overall RTOP values decrease. Again for 100 samples, the profile flattens out.

Application to in-vivo Mouse Acquisition Finally, we apply our method to in vivo acquired data from a C57Bl6 wild-type mouse. The results are shown in Fig. 4. First, we estimate MSD and RTOP for the whole data and show their values for different diffusion times on the top left. RTOP decreases as time increases, which corresponds with the in-silico experiments. In MSD we first find an overall increase, after which a small decrease is seen. The latter phenomenon does not correspond with what we previously found. We then again randomly subsample the data for all voxels in the ROI and estimate the MSE, together with the MSD and RTOP for a chosen diffusion time of $\tau=14 \mathrm{~ms}$. The trends for all markers correspond with the synthetic data: MSE increases, RTOP decreases, and MSD stays the same as the number of samples decreases.

\section{Discussion and Conclusion}

In this work, we proposed a novel functional basis to efficiently represent the multi-spherical diffusion signal over both three-dimensional q-space and diffusion time. We regularized this basis by imposing both smoothness in the fitted signal using Laplacian regularization and sparsity in the fitted coefficients. Compared to the work by Fick et al. [4], the main methodological differences are the qspace representation, where we use the MAP basis instead of 3D-SHORE, and the sparsity term. As Figure $2 \mathrm{~b}$ shows, using MAP allows us to fit the multispherical signal better than [4] using the same number of coefficients. We remark 


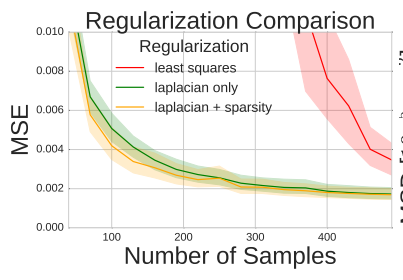

(a) Effect on MSE

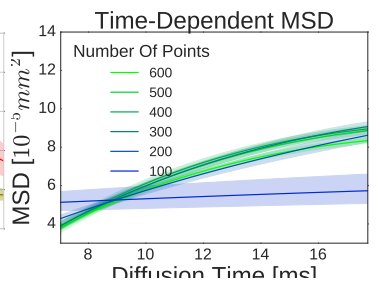

(b) Effect on MSD

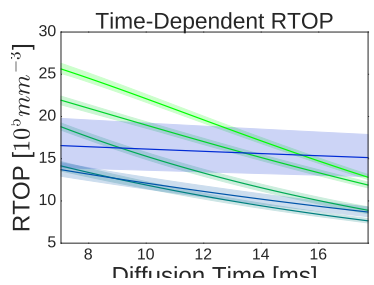

(c) Effect on RTOP

Fig. 3: Effect of random subsampling at $\mathrm{SNR}=20$ on (a) mean squared error (MSE) for different regularization techniques, (b) the time-dependent ReturnTo-Origin Probability (RTOP) and (c) Mean Squared Displacement (MSD). (a) Our combined sparsity and Laplacian regularization (yellow) has lower MSE than only Laplacian (green) and least squares (red). (b) and (c) show the MSD and RTOP using 600 samples (green) to 100 samples (blue).

that DTI fits the multi-spherical signal worst as it cannot describe diffusion restriction over $\mathbf{q}$ or $\tau$. This limitation becomes more apparent at higher bvalues, which is exactly where the diffusion signal is most characterizing of the underlying tissue.

This work is also the first to estimate and study the progress of threedimensional $\mathbf{q}$-space indices over diffusion time. Our basis is especially wellsuited for this exploration. For any evaluated diffusion time the basis reduces to MAP, which allows us to calculate all of its previously proposed indices [12, 13]. For now, we focused on the well-known Mean Squared Displacement (MSD) and Return-to-Origin Probability (RTOP). We found that the recovered trends in synthetic data correspond with what we expect from theory (Figs. 3b,3c). As diffusion time increases, spins get more time to diffusive, so MSD increases and RTOP decreases. Decreasing the number of samples did not influence MSD trends so much, but RTOP trends did lower, possibly related to removal of samples along the "restricted" direction in the signal. Overall, a lower bound of reliable index estimation seems to be around 200 samples using random subsampling, as both profiles flatten out at this point.

Applying our method to real multi-spherical data from a mouse produces mostly coherent results with the simulated data. Again we find that RTOP drops as diffusion time increases, and lowering the number of samples decreases the RTOP and leaves MSD mostly unaffected. As fewer samples were used, we found more negative (infeasible) RTOP values. To avoid this, our framework could still be improved by adding a positivity constraint like in Özarslan et al. [12].

Regardless, our multi-spherical basis is the first of its kind in being specifically designed to represent the four-dimensional EAP and analyzing its properties. Our proposed regularization allows us to significantly reduce the number of measured samples, which may eventually bring multi-spherical diffusion MRI within the reach of clinical application. 

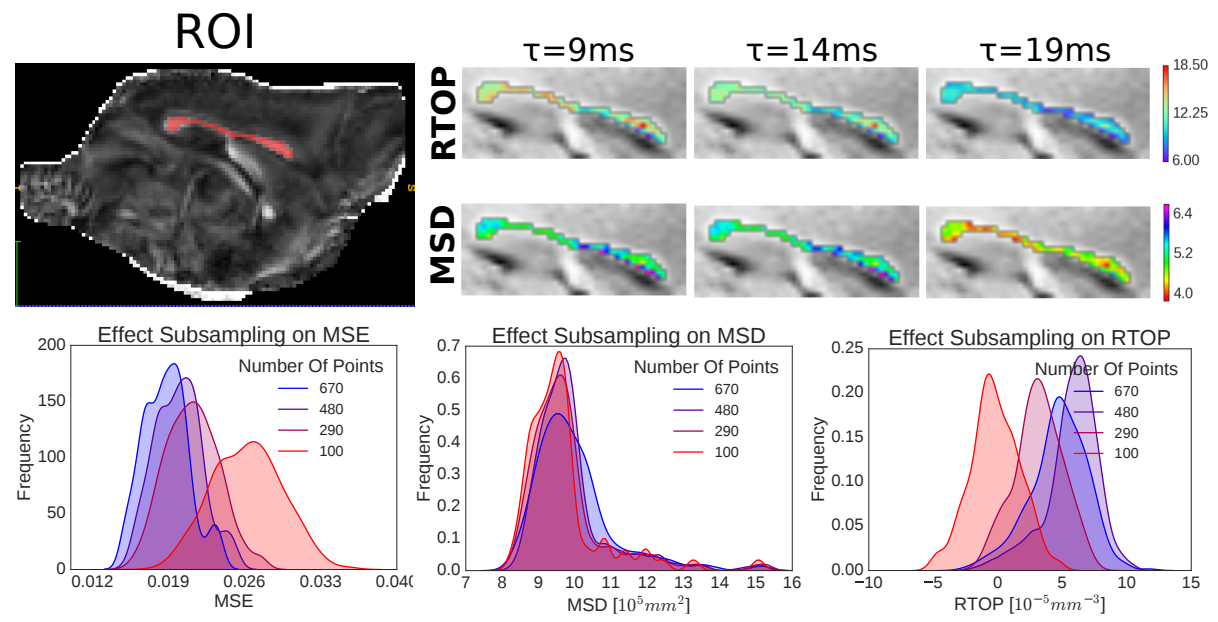

Fig. 4: (top-left) Region of interest in mouse corpus callosum. (top-right) Maps of RTOP and MSD for different diffusion times. (bottom) Histograms of the MSE (left), MSD (middle) and RTOP (right) for different numbers of fitted points. The RTOP and MSD were calculated for $\tau=14 \mathrm{~ms}$.

\section{Acknowledgments}

This work was partly supported by ANR "MOSIFAH" under ANR-13-MONU0009-01, the ERC under the European Union's Horizon 2020 research and innovation program (ERC Advanced Grant agreement No 694665:CoBCoM), MAXIMS grant funded by ICM's The Big Brain Theory Program and ANR-10-IAIHU-06.

\section{A Analytic Laplacian Regularization}

We provide the analytic form of the Laplacian regularization matrix in Eq. (9). As our basis is separable in $\mathbf{q}$ and $\tau$, the Laplacian of our basis function $\Xi_{i}$ is

$$
\nabla^{2} \Xi_{i}\left(\mathbf{q}, \tau, u_{s}, u_{t}\right)=\left(\nabla_{\mathbf{q}}^{2} \Phi_{i}\left(\mathbf{q}, u_{s}\right)\right) T_{i}\left(\tau, u_{t}\right)+\Phi_{i}\left(\mathbf{q}, u_{s}\right)\left(\nabla_{\tau}^{2} T_{i}\left(\tau, u_{t}\right)\right)
$$

with $\nabla_{\mathbf{q}}^{2}$ and $\nabla_{\tau}^{2}$ the Laplacian to either $\mathbf{q}$ or $\tau$. We then rewrite Eq. (9) as

$$
\begin{aligned}
\mathbf{U}_{i k} & =\int_{\mathbb{R}}\left(\nabla_{\mathbf{q}}^{2} \Phi_{i}\right)\left(\nabla_{\mathbf{q}}^{2} \Phi_{k}\right) d \mathbf{q} \int_{\mathbb{R}} T_{i} T_{k} d \tau+\int_{\mathbb{R}} \Phi_{i} \Phi_{k} d \mathbf{q} \int_{\mathbb{R}}\left(\nabla_{\tau}^{2} T_{i}\right)\left(\nabla_{\tau}^{2} T_{k}\right) d \tau \\
& +\int_{\mathbb{R}}\left(\nabla_{\mathbf{q}}^{2} \Phi_{i}\right) \Phi_{k} d \mathbf{q}\left(\int_{\mathbb{R}} T_{i}\left(\nabla_{\tau}^{2} T_{k}\right) d \tau+\int_{\mathbb{R}}\left(\nabla_{\tau}^{2} T_{i}\right) T_{k} d \tau\right)
\end{aligned}
$$

Eq. (13) can be calculated to a closed form using the orthogonality of physicists' Hermite polynomials with respect to weighting function $e^{-x^{2}}$ on $[-\infty, \infty]$. Let us first consider the integrals with respect to $\mathbf{q}$, which all parts of the Laplacian 
regularization functional of the MAP basis [13]. Writing the second order derivative as a double apostrophe ", the Laplacian of the spatial basis is given in terms of the 1D-SHORE functions as $\nabla_{\mathbf{q}}^{2} \Phi_{i}=\phi_{n_{x}}^{\prime \prime} \phi_{n_{y}} \phi_{n_{z}}+\phi_{n_{x}} \phi_{n_{y}}^{\prime \prime} \phi_{n_{z}}+\phi_{n_{x}} \phi_{n_{y}} \phi_{n_{z}}^{\prime \prime}$. The integral of the product of two Laplacians therefore becomes a sum of 9 terms, but can be described using the following three equations:

$$
\begin{aligned}
\mathrm{U}_{n}^{m}(u) & =\int_{\mathbb{R}} \phi_{n}^{\prime \prime} \phi_{m}^{\prime \prime} d \mathbf{q}=u^{3} 2(-1)^{n} \pi^{7 / 2}\left(\delta_{n}^{m} 3\left(2 n^{2}+2 n+1\right)+\delta_{n}^{m+4} \sqrt{n ! / m !}\right. \\
& \left.+\delta_{n+2}^{m}(6+4 n) \sqrt{m ! / n !}+\delta_{n+4}^{m} \sqrt{m ! / n !}+\delta_{n}^{m+2}(6+4 m) \sqrt{n ! / m !}\right) \\
\mathrm{V}_{n}^{m}(u) & =\int_{\mathbb{R}} \phi_{n}^{\prime \prime} \phi_{m} d \mathbf{q}=u(-1)^{n+1} \pi^{3 / 2}\left(\delta_{n}^{m}(1+2 n) \quad+\delta_{n}^{m+2} \sqrt{n(n-1)}+\delta_{n+2}^{m} \sqrt{m(m-1)}\right)
\end{aligned}
$$

$\mathrm{W}_{n}^{m}(u)=\int_{\mathbb{R}} \phi_{n} \phi_{m} d \mathbf{q}=u^{-1} \delta_{n}^{m}(-1)^{n} /\left(2 \pi^{1 / 2}\right)$

Using the functions in Eq. (14) we define the q-dependent parts of Eq. (13):

$$
\begin{aligned}
& \int_{\mathbb{R}}\left(\nabla_{\mathbf{q}}^{2} \Phi_{i}\right)\left(\nabla_{\mathbf{q}}^{2} \Phi_{k}\right) d \mathbf{q}= \frac{u_{x}^{3}}{u_{y} u_{z}} \mathrm{U}_{x_{i}}^{x_{k}} \mathrm{~W}_{y_{i}}^{y_{k}} \mathrm{~W}_{z_{i}}^{z_{k}}+2 \frac{u_{x} u_{y}}{u_{z}} \mathrm{~V}_{x_{i}}^{x_{k}} \mathrm{~V}_{y_{i}}^{y_{k}} \mathrm{~W}_{z_{i}}^{z_{k}}+\frac{u_{y}^{3}}{u_{z} u_{x}} \mathrm{U}_{y_{i}}^{y_{k}} \mathrm{~W}_{z_{i}}^{z_{k}} \mathrm{~W}_{x_{i}}^{x_{k}} \\
&+2 \frac{u_{y} u_{z}}{u_{x}} \mathrm{~V}_{y_{i}}^{y_{k}} \mathrm{~V}_{z_{i}}^{z_{k}} \mathrm{~W}_{x_{i}}^{x_{k}}+\frac{u_{z}^{3}}{u_{x} u_{y}} \mathrm{U}_{z_{i}}^{z_{k}} \mathrm{~W}_{x_{i}}^{x_{k}} \mathrm{~W}_{y_{i}}^{y_{k}}+2 \frac{u_{x} u_{z}}{u_{y}} \mathrm{~V}_{x_{i}}^{x_{k}} \mathrm{~V}_{z_{i}}^{z_{k}} \mathrm{~W}_{y_{i}}^{y_{k}} \\
& \int_{\mathbb{R}}\left(\nabla_{\mathbf{q}}^{2} \Phi_{i}\right)\left(\Phi_{k}\right) d \mathbf{q}=\frac{u_{x}}{u_{y} u_{z}} \mathrm{~V}_{x_{i}}^{x_{k}} \mathrm{~W}_{y_{i}}^{y_{k}} \mathrm{~W}_{z_{i}}^{z_{k}}+\frac{u_{y}}{u_{x} u_{z}} \mathrm{~V}_{x_{i}}^{x_{k}} \mathrm{~W}_{y_{i}}^{y_{k}} \mathrm{~W}_{z_{i}}^{z_{k}}+\frac{u_{z}}{u_{x} u_{y}} \mathrm{~V}_{x_{i}}^{x_{k}} \mathrm{~W}_{y_{i}}^{y_{k}} \mathrm{~W}_{z_{i}}^{z_{k}} \\
& \int_{\mathbb{R}} \Phi_{i} \Phi_{k} d \mathbf{q}=\frac{1}{u_{x} u_{y} u_{z}} \mathrm{~W}_{x_{i}}^{x_{k}} \mathrm{~W}_{y_{i}}^{y_{k}} \mathrm{~W}_{z_{i}}^{z_{k}}
\end{aligned}
$$

For terms with $\tau$, we denote the operator $M_{x_{1}}^{x_{2}}=\min \left(x_{1}, x_{2}\right)$ for the minimal value of $x_{1}, x_{2}$ and $H_{x}$ the Heaviside step function with $H_{x}=1$ iff $x \geq 0$.

$$
\begin{aligned}
& \int_{\mathbb{R}}\left(\nabla_{\tau}^{2} T_{i}\right)\left(\nabla_{\tau}^{2} T_{k}\right) d \tau=\left(\frac{1}{4}|o(i)-o(k)|+\frac{1}{16} \delta_{o(i)}^{o(k)}+M_{o(i)}^{o(k)}\right. \\
&+\sum_{p=1}^{M_{o(i)}^{o(k)}+1}(o(i)-p)(o(k)-p) H_{M_{o(i)}^{o(k)}-p}+H_{o(i)-1} H_{o(k)-1}(o(i)+o(k)-2 \\
&\left.\left.+\sum_{p=0}^{M_{o(i)-1}^{o(k)-2}} p+\sum_{p=0}^{M_{o(i)-2}^{o(k)-1}} p+M_{o(i)-1}^{o(k)-1}(|o(i)-o(k)|-1) H_{(|o(i)-o(k)|-1)}\right)\right) \\
&\left(\int_{\mathbb{R}} T_{i}\left(\nabla_{\tau}^{2} T_{k}\right) d \tau+\int_{\mathbb{R}}\left(\nabla_{\tau}^{2} T_{i}\right) T_{k} d \tau\right)=u_{t}\left(\frac{1}{2} \delta_{o(i)}^{o(k)}+\left(1-\delta_{o(i)}^{o(k)}\right) \cdot|o(i)-o(k)|\right) \\
& \int_{\mathbb{R}} T_{i} T_{k} d \tau=1 / u_{t} \delta_{o(k)}^{o(i)}
\end{aligned}
$$

\section{References}

1. Novikov, Dmitry S., et al. "Revealing mesoscopic structural universality with diffusion." PNAS 111.14 (2014): 5088-5093. 
2. Burcaw, Lauren M. et al., "Mesoscopic structure of neuronal tracts from timedependent diffusion." NeuroImage 114 (2015): 18-37.

3. De Santis, Silvia et al., "Including diffusion time dependence in the extra-axonal space improves in vivo estimates of axonal diameter and density in human white matter." NeuroImage 130 (2016): 91-103.

4. Fick, Rutger, et al. "A unifying framework for spatial and temporal diffusion in diffusion MRI." IPMI. Springer International Publishing, 2015.

5. Candès, Emmanuel J., and Michael B. Wakin. "An introduction to compressive sampling." IEEE Signal Proc Mag 25.2 (2008): 21-30.

6. Candès, Emmanuel J. et al. "Robust uncertainty principles: Exact signal reconstruction from highly incomplete frequency information." IEEE T Inform Theory 52.2 (2006): 489-509.

7. Paquette, Michael, et al. "Comparison of sampling strategies and sparsifying transforms to improve compressed sensing diffusion spectrum imaging." MRM 73.1 (2015): 401-416.

8. Merlet, Sylvain L., and Rachid Deriche. "Continuous diffusion signal, EAP and ODF estimation via compressive sensing in diffusion MRI." MIA 17.5 (2013): 556-572.

9. Rathi, Yogesh, et al. "Multi-shell diffusion signal recovery from sparse measurements." MIA 18.7 (2014): 1143-1156.

10. Bilgic, Berkin, et al. "Accelerated diffusion spectrum imaging with compressed sensing using adaptive dictionaries." MRM 68.6 (2012): 1747-1754.

11. Stejskal, E. O. "Use of spin echoes in a pulsed magneticfield gradient to study anisotropic, restricted diffusion and flow." J Chem Phys 43.10 (1965): 3597-3603.

12. Özarslan, Evren, et al. "Mean apparent propagator (MAP) MRI: a novel diffusion imaging method for mapping tissue microstructure." NeuroImage 78 (2013): 16-32.

13. Fick, Rutger HJ, et al. "MAPL: Tissue microstructure estimation using Laplacianregularized MAP-MRI and its application to HCP data." NeuroImage 134 (2016): 365-385.

14. Hosseinbor, A. Pasha, et al. "Bessel fourier orientation reconstruction (bfor): An analytical diffusion propagator reconstruction for hybrid diffusion imaging and computation of q-space indices." NeuroImage 64 (2013): 650-670.

15. Özarslan, Evren, et al. "Nuclear magnetic resonance characterization of general compartment size distributions." New J Phys 13.1 (2011): 015010.

16. Callaghan, Paul T. "Pulsed-gradient spin-echo NMR for planar, cylindrical, and spherical pores under conditions of wall relaxation." J Magn Reson, Series A 113.1 (1995): 53-59.

17. Steven Diamond and Stephen Boyd "CVXPY: A Python-Embedded Modeling Language for Convex Optimization", J Mach Learn Res 17.83 (2016): 1-5

18. Garyfallidis, Eleftherios, et al. "Dipy, a library for the analysis of diffusion MRI data." Frontiers in neuroinformatics 8 (2014): 8.

19. P. A. Cook et al., "Camino: Open-Source Diffusion-MRI Reconstruction and Processing", 14th ISMRM, Seattle, WA, USA, p. 2759, May 2006.

20. Aboitiz, Francisco et al. "Fiber composition of the human corpus callosum." Brain Res 598.1 (1992): 143-153.

21. Lamantia, AS. and P. Rakic. "Cytological and quantitative characteristics of four cerebral commissures in the rhesus monkey." J Comp Neurol 291.4 (1990): 520-537.

22. Alexander, Daniel C., et al. "Orientationally invariant indices of axon diameter and density from diffusion MRI." NeuroImage 52.4 (2010): 1374-1389.

23. Jesper L.R. Andersson and Stamatios N. Sotiropoulos. An integrated approach to correction for off-resonance effects and subject movement in diffusion MR imaging. NeuroImage, 125:1063-1078, 2016. 